\title{
Lempel-Ziv complexity in schizophrenia: A MEG study
}

\author{
Alberto Fernández ${ }^{\mathrm{a}, \mathrm{b}, *}$, María-Inés López-Ibor ${ }^{\mathrm{a}}$, Agustín Turrero ${ }^{\mathrm{c}}$, Juan-Matías Santos ${ }^{\mathrm{a}}$, \\ María-Dolores Morón ${ }^{\mathrm{d}}$, Roberto Hornero ${ }^{\mathrm{e}}$, Carlos Gómez ${ }^{\mathrm{e}}$, María Andreina Méndez ${ }^{\mathrm{a}}$, Tomás Ortiz ${ }^{\mathrm{a}}$, \\ Juan José López-Ibor ${ }^{\mathrm{a}, \mathrm{d}}$
}

a Department of Psychiatry and Psychological Medicine, Complutense University, Madrid, Spain

${ }^{\mathrm{b}}$ Laboratory of Cognitive and Computational Neuroscience, Centre for Biomedical Technology (CTB), Technical University and Complutense University, Madrid, Spain

${ }^{c}$ Department of Statistics and Operative Research, Complutense University, Madrid, Spain

${ }^{\mathrm{d}}$ Institute of Psychiatry and Mental Health, San Carlos University Hospital, Madrid, Spain

${ }^{\mathrm{e}}$ Biomedical Engineering Group, University of Valladolid, Spain

Keywords:

Schizophrenia

Lempel-Ziv complexity

Magnetoencephalography

Neurodevelopmental

Neurodegenerative
H I G H L I G H T S

- Schizophrenic patients show higher complexity values as compared to controls. - Schizophrenic patients showed a tendency to reduced complexity values as a function of age while controls showed the opposite tendency. - The tendency observed in schizophrenic patients parallels the tendency observed in Alzheimer disease patients.

\begin{abstract}
A B S T R A C T
Objective: The neurodevelopmental-neurodegenerative debate is a basic issue in the field of the neuropathological basis of schizophrenia (SCH). Neurophysiological techniques have been scarcely involved in such debate, but nonlinear analysis methods may contribute to it.

Methods: Fifteen patients (age range 23-42 years) matching DSM IV-TR criteria for SCH, and 15 sex- and age-matched control subjects (age range 23-42 years) underwent a resting-state magnetoencephalographic evaluation and Lempel-Ziv complexity (LZC) scores were calculated.

Results: Regression analyses indicated that LZC values were strongly dependent on age. Complexity scores increased as a function of age in controls, while SCH patients exhibited a progressive reduction of LZC values. A logistic model including LZC scores, age and the interaction of both variables allowed the classification of patients and controls with high sensitivity and specificity.

Conclusions: Results demonstrated that SCH patients failed to follow the "normal" process of complexity increase as a function of age. In addition, SCH patients exhibited a significant reduction of complexity scores as a function of age, thus paralleling the pattern observed in neurodegenerative diseases.

Significance: Our results support the notion of a progressive defect in $\mathrm{SCH}$, which does not contradict the existence of a basic neurodevelopmental alteration.
\end{abstract}

\section{Introduction}

Schizophrenia $(\mathrm{SCH})$ is a serious psychiatric disorder with a range of "positive" symptoms including paranoia, delusions and hallucinations, as well as "negative" symptoms such as cognitive impairment, flattened affect and disorganised thinking. Symptoms most commonly present in late adolesence in males and somewhat later in females, and the first psychotic episode is commonly pre- ceded by a "prodromal" phase of progressively more severe symptoms which can last months or years.

Clinically, it is a common observation that a functional decline starts around the time of the late prodromal phase or around the first psychotic episode (Tully and McGlashan, 2006), suggesting that a neurodegenerative process may be at the core of SCH. However, this neurodegenerative perspective has been challenged by the so-called neurodevelopmental theory (Murray and Lewis, 1987; Rapoport et al., 2005) which considers SCH as the product of a deviation in neurodevelopmental processes that occurs before the onset of clinical symptoms (Rapoport et al., 2005). The neurodevelopmental vs. neurodegenerative debate is therefore a basic issue in the biology of $\mathrm{SCH}$. 
Magnetoencephalography (MEG) has been widely used in $\mathrm{SCH}$ research. MEG studies have replicated the well-known pattern of fronto-temporal low-frequency activity observed in EEG, and have also demonstrated abnormal sensory gating and basic cognitive processing (Canive et al., 1996; Kissler et al., 2000; Fehr et al., 2003; Wienbruch et al., 2003; Edgar et al., 2005; Lopez-Ibor et al., 2008; Naatanen and Kahkonen, 2009). A further step in this research is non-linear dynamics analysis (NDA). Complexity analysis is a particular form of NDA as applied to human brain activity, e.g. as measured using electroencephalography (EEG) or magnetoencephalography (MEG). EEG-MEG complexity analyses usually measure the regularity/predictability of brain oscillations and/or attempt to estimate the number of independent oscillators underlying the observed signal (Lutzenberger et al., 1995; Jeong et al., 1998). Numerous complexity measures have been proposed (see Tononi et al., 1998) including: dimensional complexity, algorithmic complexity, neural complexity, fractal dimension, first Lyapunov component, correlation dimension, Lempel-Ziv Complexity (LZC), etc. (Elbert et al., 1992; Koukkou et al., 1993; Jeong et al., 1998; Kim et al., 2000; Kotini and Anninos, 2002; Li et al., 2008). For example, the dimensional complexity refers to the classical method of the so-called correlation dimension. The algorithmic or Kolmogorov complexity is defined as the length of the string shortest description in some fixed description language. Finally, the neural complexity is a statistical measure that captures regularities based on the deviation from independence among subsets of a system. In our study we have used the LZC, a measure for finite sequences that assigns larger values to more complex data (Lempel and Ziv, 1976). LZC is an appropriate measure of complexity in Kolmogorov's sense.

Previous studies of neural activity complexity have provided somewhat contradictory results, with increased or decreased complexity values seen in SCH patients, depending upon the characteristics of the patient sample and the particular complexity parameter utilised. However, many studies revealed a pattern of increased complexity values, especially in frontal areas, in $\mathrm{SCH}$ patients (Elbert et al., 1992; Saito et al., 1998; Na et al., 2002; Irisawa et al., 2006; Li et al., 2008). This may be related to the increased "irregularity" of SCH patients' behaviour (Koukkou et al., 1993).

Interestingly, according to Anokhin et al. (1996) and Meyer-Lindenberg (1996), there is an association between brain maturation and complexity scores, with a brisk increase of EEG complexity from childhood to early adolescence, and a further sustained increase from adolescence to late adulthood. Thus "normal" brain development seems to be characterised by an increase of complexity scores, at least until early senescence (Fernández et al., 2010).

However, Fernández et al. (2009) found that this normal pattern of increasing complexity with age is absent in attention deficithyperactivity disorder (ADHD), a disease with clear neurodevelopmental components (Shaw et al., 2007). In order to further investigate the possible involvement of abnormal complexity in neurodevelopmental disorders, we therefore decided to measure LZC scores in a group of SCH patients and healthy controls. Our objective was twofold; first we tested a potentially abnormal pattern of complexity evolution as a function of age in $\mathrm{SCH}$ patients. Subsequently, the pattern of increased complexity in $\mathrm{SCH}$ was tested.

\section{Methods}

MEG epochs were analysed by means of LZC. LZC is a measure of finite sequences and is essentially a measure of the number of distinct substrings and the rate of their occurrence along the sequence (Lempel and Ziv, 1976). (Aboy et al. (2006) investigated the factors that affects $L Z C$ and concluded that $L Z C$ represents an estimate of the number of different frequency components that actually compose the brain signals. See the Appendix A for details of the analysis.

\subsection{Subjects}

Fifteen right-handed patients receiving care at the San Carlos University Hospital Institute of Psychiatry and Mental Health, who fulfilled the DSM-IV diagnostic criteria for $\mathrm{SCH}$, were included in the study. Diagnosis was made with the Spanish version of the SCID-I (First et al., 1997). In order to obtain a homogeneous sample, we only included patients exhibiting a high degree of positive psychotic symptoms. The Scale for the Assessment of Positive Symptoms (SAPS) (Andreasen, 1984) was used to evaluate positive symptoms of schizophrenia. According to a previous study (Lopez-Ibor et al., 2008), a minimum score of 70 (of a maximum of 165 ), and a minimal score of 29 (of maximum of 65) in the delusional activity subscale, were required to enter in the study.

The control group consisted of fifteen right-handed sex- and age-matched control subjects with no history of psychiatric disorder (Table 1). Subjects with a history of neurological diseases, head trauma, or drug abuse were excluded from the study. Consequently, the data set consists of 30 sex- and age- jointly matched case-control pairs.

Patients matching the above described criteria were consecutively submitted to MEG scans. At the time of the study, all of the schizophrenic patients were using atypical antipsychotic medication. In addition, two were on the typical antipsychotic haloperidol. Table 1 shows the dose equivalent of the medications and the duration of the illness. Prior to MEG recording, all subjects provided informed consent after being informed as to the technical and ethical considerations of the research. The study was approved by the ethics committee of the San Carlos University Hospital in Madrid. This sample has been partially described elsewhere (Lopez-Ibor et al., 2008).

\subsection{Data collection}

MEG recordings were acquired with a 148-channel whole-head magnetometer (MAGNES $2500 \mathrm{WH}^{\mathrm{(} \mathrm{k}}$, 4D Neuroimaging, San Diego, CA) placed in a magnetically shielded room at "Centro de Magnetoencefalografía Dr. Pérez-Modrego" (Madrid, Spain). Subjects were in an awake but resting state with their eyes closed and under supervision during the recording. They were asked to avoid blinking and making movements. For each subject, five minutes of MEG signal were acquired at a sampling frequency of $678.17 \mathrm{~Hz}$ using a hardware band-pass filter of $0.1-200 \mathrm{~Hz}$. Afterwards, these recordings were down-sampled by a factor of $4(169.549 \mathrm{~Hz}, 50863 \mathrm{sam}$ ples). This process consisted of filtering the data to avoid aliasing (Nyquist criterion) and downsampling the recordings. The anti-aliasing filter was a second-order Butterworth IIR routine applied to the signals in both forward and reverse directions to avoid net phase shift with cut-off frequency at $76.30 \mathrm{~Hz}(45 \%$ of the final sample rate: $169.549 \mathrm{~Hz}$ ).

Artifact-free epochs of $20 \mathrm{~s}$ were selected off-line by a technician who was blind to diagnosis (mean $=13.7$ artifact-free epochs per channel and subject). Of note, no significant differences in terms of artifact-free epochs were found between patients and controls. Finally, all epochs were filtered between 1.5 and $40 \mathrm{~Hz}$, and copied to a computer as ASCII files for further complexity analysis.

\subsection{Lempel-Ziv complexity calculation}

LZC analysis is based on a coarse-graining of measurements. Therefore, the MEG signal must be previously transformed into a 
Table 1

Characteristics of the sample (mean \pm s.d.; min-max where applicable), n.a.: not applicable.

\begin{tabular}{lll}
\hline & Patients & Controls \\
\hline Sex & $15 ; 11 \mathrm{M}, 4 \mathrm{~F}$ & $15 ; 11 \mathrm{M}, 4 \mathrm{~F}$ \\
Age (years) & $31.93 \pm 6.60 ; 23-42$ & $31.87 \pm 6.02 ; 23-$ \\
& & 42 \\
Length of illness (years) & $5.58 \pm 3.20 ; 1-9$ & n.a. \\
$\begin{array}{l}\text { Haloperidol equivalent dose } \\
\quad 33.00 \pm 19.02 ; 7-62\end{array}$ & n.a. \\
$\quad$ Lorazepam equivalent dose & $1.74 \pm 1.70 ; 0-4.5$ & n.a. \\
$\quad$ (mg) & $89.38 \pm 13.71 ; 72.00-$ & n.a. \\
SAPS scores Total & 117.0 & \\
$\quad$ Delusions & $40.77 \pm 8.09 ; 29-51$ & \\
& &
\end{tabular}

finite symbol string. In this study we used the simplest possible way: a binary sequence conversion (zeros and ones). By comparison with a threshold $T d$, the original signal samples are converted into a $0-1$ sequence $P=s(1), s(2), \ldots, s(n)$, with $s(i)$ defined by (Zhang et al., 2001):

$s(i)= \begin{cases}0 & \text { if } x(i)<T_{d} \\ 1 & \text { if } x(i) \geqslant T_{d}\end{cases}$

The threshold $T d$ is estimated as the median value of the signals amplitude in each channel. We selected the median value because it is more robust to outliers (Nagarajan, 2002; Zhang et al., 2001). The string $P$ is then scanned from left to right and a complexity counter $c(n)$ is increased by one unit every time a new subsequence of consecutive characters is encountered in the scanning process. The detailed algorithm for the measure of the $L Z$ complexity is included in Apendix A.

\subsection{Data reduction}

A LZC-normalised score was obtained for each channel and participant, calculating the mean value over all the 20 -s artifact-free epochs. Thus there were a total of 148 LZC scores per subject available for statistical analysis. In order to avoid the multiple-comparisons problems posed by this amount of data, the 148 channels were grouped into five regions (anterior, central, left lateral, right lateral, and posterior), and the mean LZC value for each region was calculated (see Fig. 1). This approach has been successfully utilised previously (Fernández et al., 2009, 2010).

\subsection{Statistical analysis}

Two-sample $t$-tests were applied to evaluate LZC differences between the groups.

Pearson correlation coefficients were used to examine the relationship among LZC scores between regions in both groups. This analysis offers a hint about a potential variable reduction process when the analysis of the prognostic importance of LZC scores is undertaken. Correlations between LZC scores and clinical variables were assessed using the Spearman's correlation coefficient.

In order to avoid the potential confounding influence of age, the relationship between age and LZC scores was investigated by means of linear regression analyses in the two groups in each of the five regions.

Logistic regression analyses were applied with "Diagnosis" as a dependent variable in order to ascertain whether neural complexity scores could act as predictors of diagnosis. The independent (or predictor) variables initially considered were the LZC scores obtained for each region. The model's performance was assessed by means of classification tables, together with standard Hosmer and Lemeshow (1989) goodness of fit tests, and the Nagelkerke

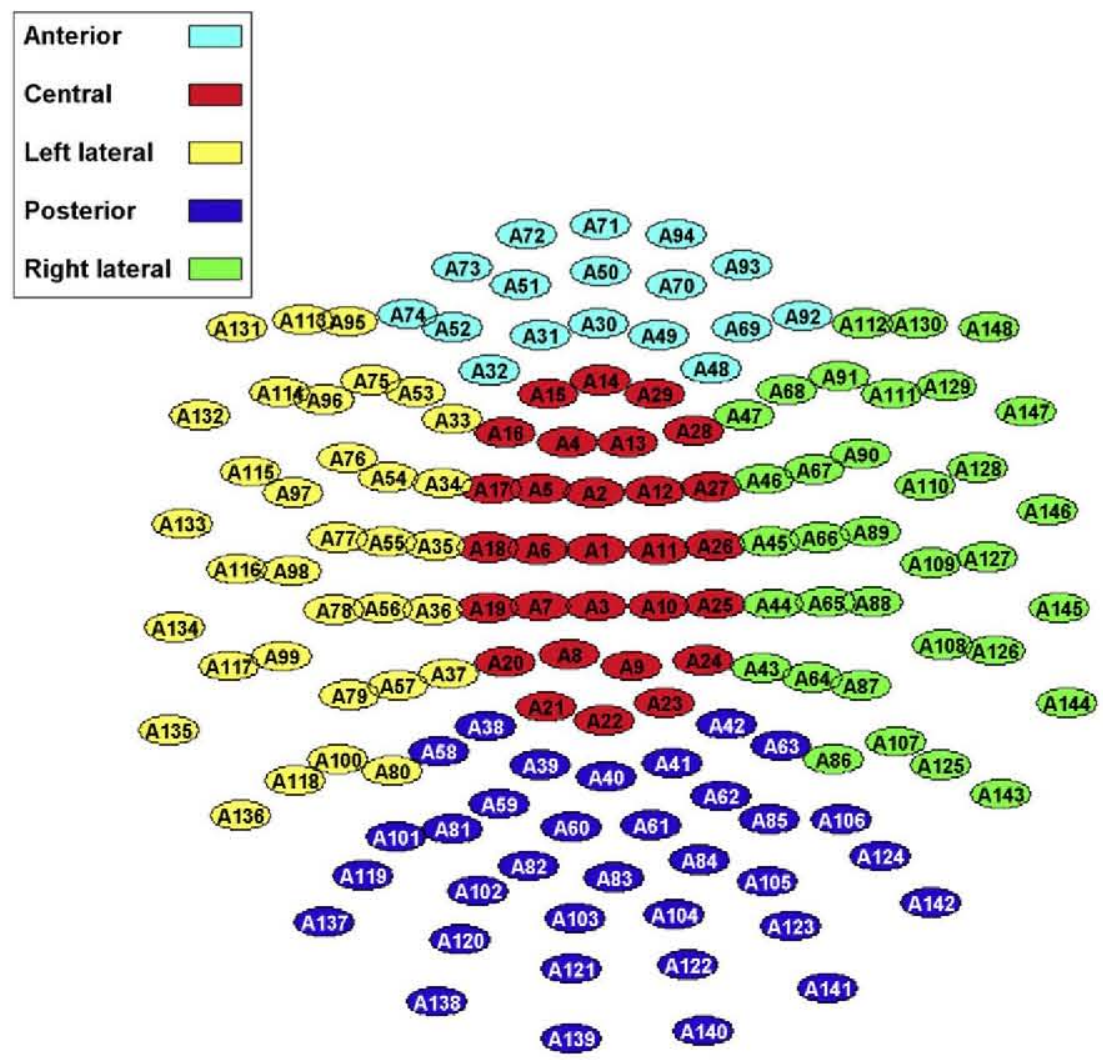

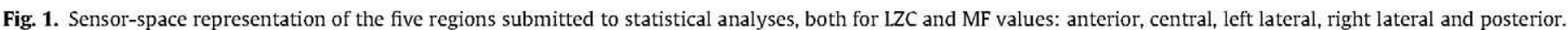


$R^{2}$ goodness of fit statistics. The efficiency of the final models was assessed by the area under the receiver operating characteristic (ROC) curve, together with the usual goodness of fit tests.

\section{Results}

\subsection{LZC differences between groups}

The means and standard deviations of the LZC scores for SCH and control groups in each of the five regions are shown in Table 2 which also shows the between-group differences in terms of Cohen's $d$ effect sizes (Cohen, 1988). SCH patients' LZC values were greater than those of the controls in all brain regions (see also Fig. 2). $T$-tests showed statistically significant $(p<0.05)$ differences in all regions with the exception of the posterior region where a close-to-significant trend was observed $(p=0.071)$. For comparison purposes, a conventional spectral analysis was also performed in order to evaluate the characteristics of MEG signals (see Fig. 3).

\subsection{Correlation analysis}

LZC scores in each region showed a strong linear correlation with the LZC scores in each other region, in both groups. All correlation coefficients, except the anterior-posterior and central-posterior correlations, were over 0.80 , with the central, anterior left lateral and right lateral regions being the most strongly correlated regions.

However, no significant non-parametric correlation was found among LZC scores and the clinical variables using non-parametric analyses: SAPS total score $(p>0.27)$, length of illness $(p>0.10)$, and medications in terms of dose equivalents, lorazepam equivalents $(p>0.31)$ and haloperidol equivalents $(p>0.61)$ (see Table 3$)$.

\subsection{Age effects}

The relationship between age and LZC scores was investigated by means of linear regression analyses in the two groups. In the SCH patients, a negative trend was observed, indicating a tendency for decreased LZC scores with increasing age in all regions. Moreover, this effect was statistically significant, according to a linear regression $t$-test, in all regions (all $p<0.008$ ), with all correlation coefficients $r$ at least -0.67 .

By contrast, in the control group, the opposite pattern emerged: LZC was positively correlated with age. This trend was clearly significant in the central ( $p=0.01$ and $r=0.641)$, anterior ( $p=0.001$ and $r=0.828$ ) and posterior ( $p=0.014$ and $r=0.620$ ) regions, and was close to significant in the right lateral $(p=0.052, r=0.511)$ and left lateral $(p=0.058, r=0.500)$ regions (see Fig. 4 ).

\subsection{Logistic regression analyses}

We fitted a series of logistic regression equations to obtain a model to discriminate between the $\mathrm{SCH}$ and control groups on the basis of their LZC scores. The dependent variable was
"Diagnosis" (schizophrenia or control). The independent (predictor) variables initially consisted of the five regional LZC scores.

In a preliminary univariate analysis, all LZC scores showed a positive predictive power (all likelihood ratio tests showed a $p<0.05$ ). In a multivariate analysis, including all LZC scores, a stepwise procedure selected the central region $\left(\chi_{1}^{2}=7.401, p=0.007\right)$ as a significant predictor for the final model.

In the light of the results discussed above regarding age, the model was refitted including age as an additional variable. This model showed no significant improvement over the previous model excluding age $\left(\chi_{1}^{2}=0.219, p=0.640\right)$, showing that age per se adds little once central LZC value has been included. This result was expected because age is a matching variable. However, Fig. 4 shows very different behaviour of LZC values as a function of age, suggesting that age acts as a modifier rather than as a confounding effect and that age should be tested in terms of an age $\times$ central LZC interaction.

The model-building process continued by ascertaining the correct scale in the Logit for age and central LZC variables. This analysis showed evidence of linearity in both cases. Finally we searched for an age $x$ central LZC interaction. The interaction was strongly significant $\left(\chi_{1}^{2}=17.331, p=0.001\right)$. Thus, age has a modifier effect. Therefore this model including central LZC score, age and the age $\times$ center LZC interaction was selected as the best model for $\mathrm{SCH}-$ Control discrimination. The Hosmer and Lemeshow statistic was 4.265 ( $p=0.832$ ) and the Nagelkerke $R^{2}$ goodness of fit statistic was 0.753 , indicating that $75.3 \%$ of the "variation" in the diagnosis (SCH vs. control) was explained by the logistic model. The area under the ROC (see Fig. 5) curve was 0.956 (95\% CI $=0.886$; 1.000 ). The sensitivity and specificity of model was $86.7 \%$ when a 0.50 cut-off point is adopted (two misclassified subject in each diagnostic group).

\section{Discussion}

Our data show that compared to healthy controls, patients with schizophrenia have more complex resting-state neural activity. Neural activity was assessed using MEG and complexity was calculated in terms of LZC scores. While SCH patients showed increased complexity across the brain, the increase was especially marked over central cortical regions.

Furthermore, we observed that LZC values are strongly dependent on age, but that the direction of the relationship was reversed in schizophrenic patients as opposed to healthy people: in controls complexity increased with age but in patients it decreased with age. A logistic model was able to predict group assignment (diagnosis) on the basis of individual LZC scores and ages. Our findings partially support prior reports (Elbert et al., 1992; Na et al., 2002; Irisawa et al., 2006; Li et al., 2008) of increased complexity values in $\mathrm{SCH}$, however unlike previous investigators we also investigated the effects of age.

Like all complexity measures, LZC is essentially an estimate of the predictability and variability of neural signals in terms of frequency components (see Introduction). However, LZC has advanta-

Table 2

Region $\times$ group comparisons of LZC values between SCH and control groups. The table includes results of $t$-tests, probabilities and effect sizes.

\begin{tabular}{|c|c|c|c|c|c|c|}
\hline & & Central & Anterior & Right lateral & Left lateral & Posterior \\
\hline \multirow[t]{2}{*}{$S Z$} & Mean & 0.7312 & 0.7198 & 0.6852 & 0.6813 & 0.6755 \\
\hline & $\mathrm{SD}$ & 0.0309 & 0.0382 & 0.0428 & 0.0400 & 0.0424 \\
\hline \multirow{2}{*}{ Control } & Mean & 0.6936 & 0.6911 & 0.6524 & 0.6503 & 0.6473 \\
\hline & SD & 0.0309 & 0.0265 & 0.0308 & 0.0306 & 0.0398 \\
\hline t-Statistic & & 2.888 & 2.387 & 2.408 & 2.385 & 1.877 \\
\hline$p$-Value & & 0.007 & 0.024 & 0.023 & 0.024 & 0.071 \\
\hline Effect sizes & & 1.217 & 0.873 & 0.879 & 0.871 & 0.686 \\
\hline
\end{tabular}




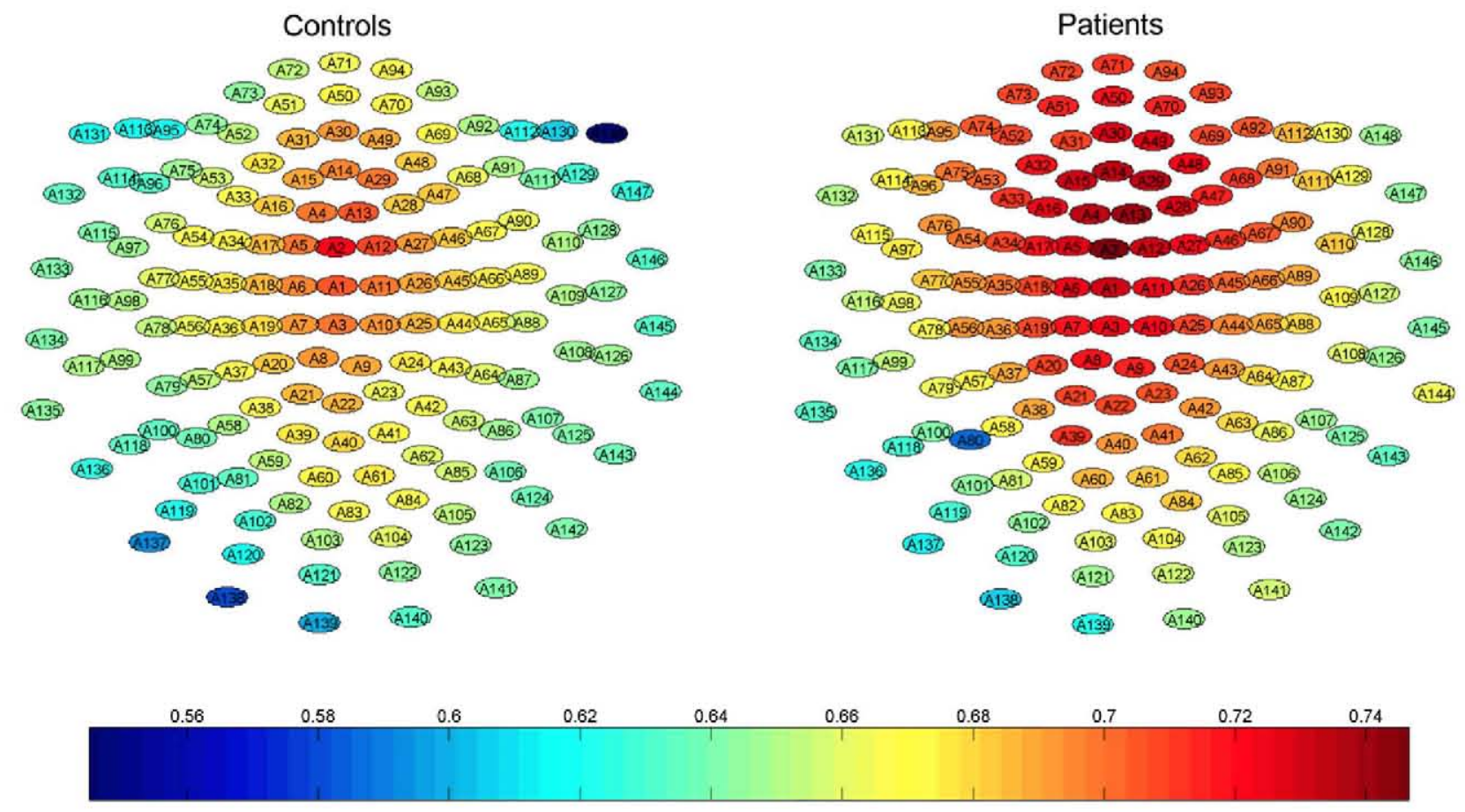

Fig. 2. Average $\mathrm{IZC}$ values in patients with $\mathrm{SCH}$ and control subjects for all channels, from A1 to A148, displayed in a colour scale. A significant increase of $\mathrm{LZC}$ values is observed in SCH. Sample size, $\mathrm{SCH}$ patients $=15$, controls $=15$. (For interpretation of the references to color in this figure legend, the reader is referred to the web version of this paper.)

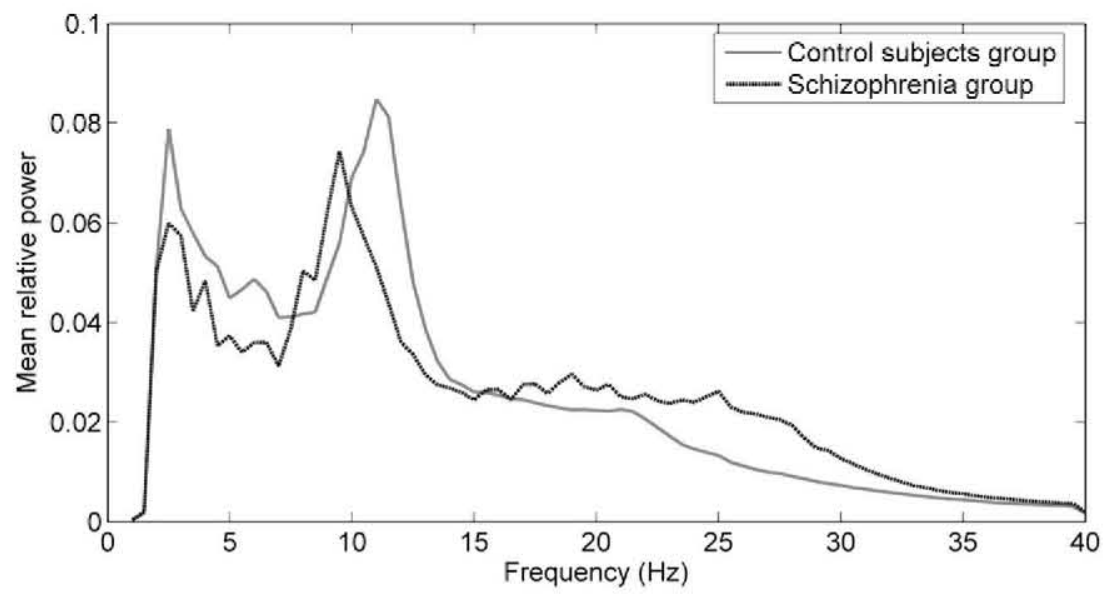

Fig. 3. Mean relative power spectra for all channels in schizophrenia and control groups. This figure illustrates the slowing of alpha peak in schizophrenia patients.

Table 3

Spearman's rank correlation coefficients, and their p-values, displaying correlations between LZC scores and the clinical variables: SAPS total score, duration of illness, and medications dose equivalent (lorazepam and haloperidol).

\begin{tabular}{|c|c|c|c|c|c|}
\hline & Central & Anterior & Right lateral & Left lateral & Posterior \\
\hline SAPS total score & $\begin{array}{l}-0.199 \\
p=0.514\end{array}$ & $\begin{array}{l}-0.329 \\
p=0.272\end{array}$ & $\begin{array}{l}-0.025 \\
p=0.936\end{array}$ & $\begin{array}{l}-0.077 \\
p=0.801\end{array}$ & $\begin{array}{l}-0.177 \\
p=0.563\end{array}$ \\
\hline Duration of illness & $\begin{array}{l}-0.293 \\
p=0.355\end{array}$ & $\begin{array}{l}0.494 \\
p=0.103\end{array}$ & $\begin{array}{l}-0.304 \\
p=0.336\end{array}$ & $\begin{array}{l}0.233 \\
p=0.467\end{array}$ & $\begin{array}{l}-0.336 \\
p=0.285\end{array}$ \\
\hline Haloperidol dose equivalent & $\begin{array}{l}-0.165 \\
p=0.609\end{array}$ & $\begin{array}{l}-0.053 \\
p=0.871\end{array}$ & $\begin{array}{l}-0.028 \\
p=0.931\end{array}$ & $\begin{array}{l}0.158 \\
p=0.625\end{array}$ & $\begin{array}{l}0.126 \\
p=0.696\end{array}$ \\
\hline
\end{tabular}

ges over traditional non-linear techniques such as the correlation dimension and the first Lyapunov exponent in terms of the analysis of MEG/EEG data. Whereas other methods require a large amount of noise-free stationary data (Eckmann and Ruelle, 1992; Grassberger and Procaccia, 1983), the LZC is robust to noise and can be applied to both deterministic and stochastic time series (Lempel and 


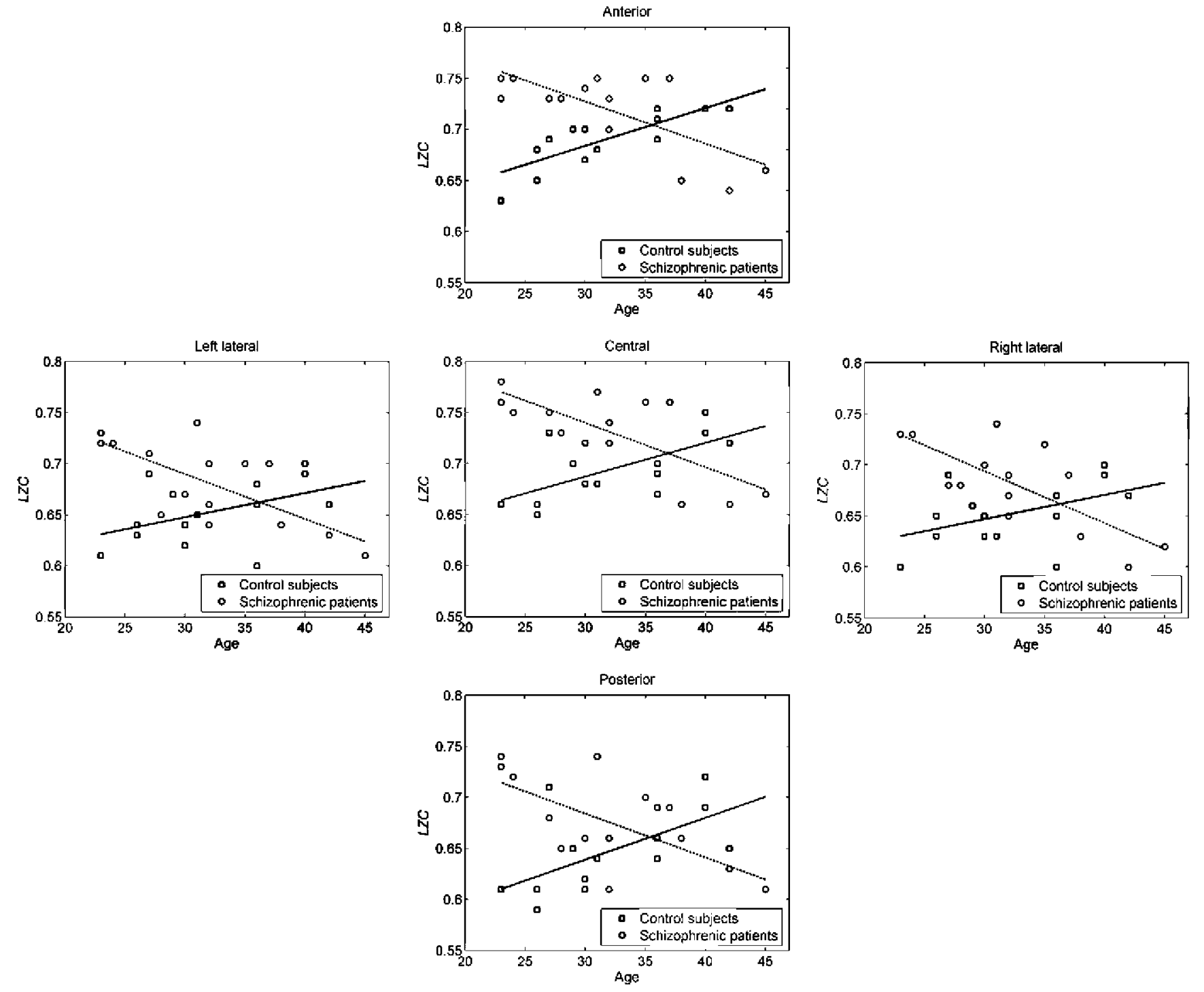

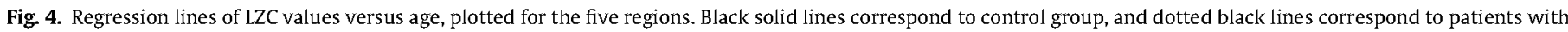

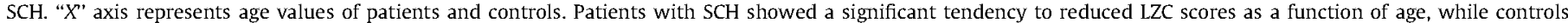
exhibited an opposite tendency. Sample size, $\mathrm{SCH}=15$, controls $=\mathbf{1 5}$.

Ziv, 1976). As the median was used as threshold for the binary conversion, LZC is also robust to outliers. Finally, Aboy et al. (2006) concluded that LZC is not affected by the power of white noise.

Early electrophysiological studies of schizophrenia emphasised the so called "dysrhythmia" (Itil, 1977) in this disease. This was later described as a pattern of, on the one hand, increased frequency variability and, also, a reduced signal amplitude (Shagass et al., 1982). It might be expected that brain signals derived from patients suffering from a disease that increases EEG-MEG variability should yield higher complexity values. In addition, the complexity of brain activity measured by EEG-MEG signals is considered to be intimately associated with the integrity of brain connectivity (Sporns et al., 2000; Jeong, 2004). Many researchers e.g. Friston $(1996,2002)$ have proposed that the fundamental pathology in $\mathrm{SCH}$ is a disconnection between functional regions of the brain, and that such dysconnectivity induces higher complexity values (Friston, 1996). See also (McGlashan and Hoffman, 2000). Dysconnectivity may lead to symptom formation and explain the altered brain response to environmental stimuli (delusions and hallucinations.) The misdistribution of connections in a system with low cognitive potential, limited reserve, and poor flexibility could be reduced by antipsychotic treatment in parallel with the attenuation of psychotic symptoms (McGlashan, 2006).

Our data are consistent with this account, but we were also able to demonstrate a novel finding, namely the divergent trajectory of complexity with age in patients and controls. Such abnormal maturational processes have also been shown in structural investigations in schizophrenia. For example, Ho et al. (2003) performed a longitudinal study in a large sample of first-episode SCH patients and controls using MRI to measure white matter, grey matter and CSF volumes. They showed a progressive enlargement of white-matter volumes, especially in the frontal lobes, in healthy controls while in the $\mathrm{SCH}$ patients the reverse was true. The authors interpreted these findings as consistent with a failure to complete the "normal" maturational process of myelination. Other studies using other methods such as diffusion tensor imaging (Jones et al., 2006) have provided similar results.

Considering these findings, and bearing in mind that the progressive loss of brain volume observed in some SCH samples is very similar to that observed in Alzheimer disease (AD) and other dementias (Krueger et al., 2010), it is difficult to maintain that schizophrenia is a purely neurodevelopmental condition. Post- 


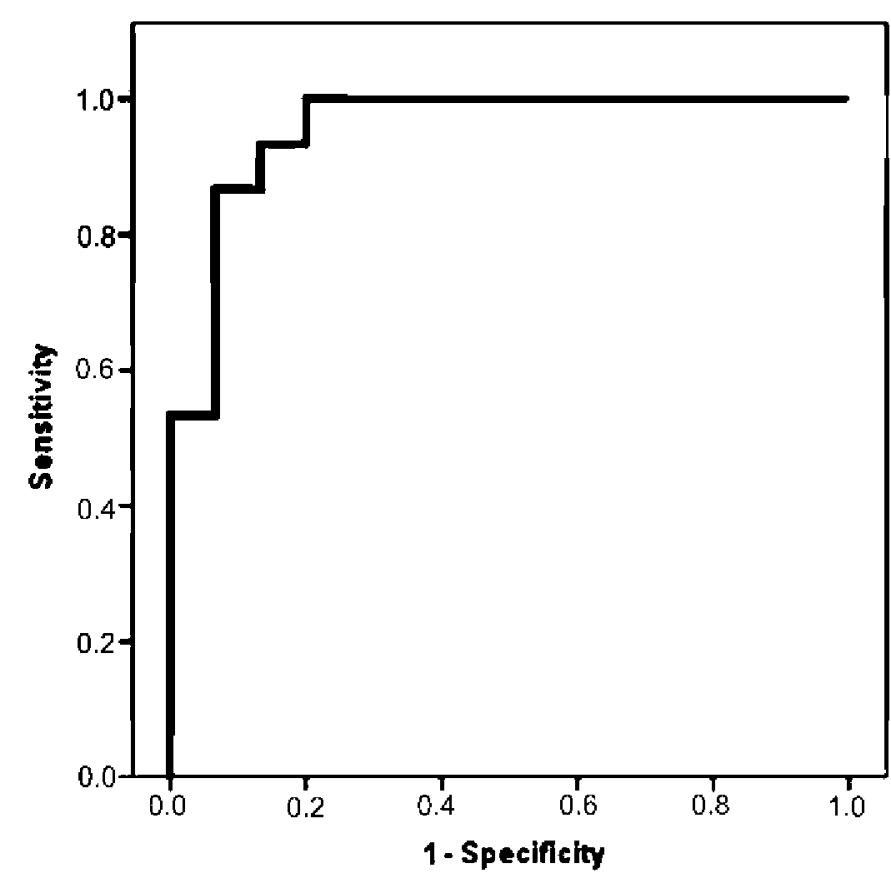

Fig. 5. ROC curve of the logistic regression model based on age and centre LZC variables.

mortem studies showing the absence of gliosis in schizophrenic brains has been considered a major argument against the idea that SCH is a neurodegenerative disease (Roberts et al., 1986, 1987) but neurodegeneration does not necessarily imply gliosis: for example excessive neuritic pruning could account for progressive brain alterations such as a progressive reduction of brain volume (Woods, 1998; Lieberman et al., 2001; Perez-Neri et al., 2006; Theberge et al., 2007).

Do our results support the alternative view of a neurodegenerative process in $\mathrm{SCH}$ ? Bearing in mind the limitations of this study (see below) it would be presumptuous to conclude that we have demonstrated a neurodegenerative process in SCH. Nevertheless, we claim our findings support a progressive defect which does not contradict the existence of a basic neurodevelopmental alteration. In a previous study we (Fernández et al., 2010) observed a progressive reduction of LZC values as a function of age in AD. The present data indicate that: 1 . SCH patients did not show the "normal" process of increasing complexity as a function of age, rather showing a reduction of complexity as a function of age, and 2. The same pattern was observed in $\mathrm{AD}$ patients.

\section{Significance}

A major limitation of our study was the fact that all patients were on medication, notably antipsychotics. Although there was no significant correlation between LZC and medication dosageequivalents, drug effects cannot be ruled out. Previously, Jeong et al. (1998) and Kim et al. (2000) likewise found no correlation between antipsychotic dose and complexity values. Nevertheless, authors were cautious when interpreting this absence of significant correlations. Higher complexity values might be still partially attributed to drug effects although similar findings of increased complexity values were obtained in non medicated samples (Saito et al., 1998).

Secondly, we utilised a cross-sectional rather than a longitudinal design to investigate age effects. A longitudinal investigation of first episode patients and young populations at risk of develop- ing SCH would be of great interest. Thirdly, our sample size was relatively small.

Notwithstanding these limitations, our data suggest that the investigation of complexity in neural activity is a promising approach to understanding the neural basis of schizophrenia and other neuropsychiatric disorders.

\section{Financial support}

This work was supported by the FUNDACIÓN MUTUA MADRILEÑA, within the project: "Estudio del procesamiento cerebral en pacientes con esquizofrenia paranoide mediante magnetoencefalografía (2004-2008)". The funding source had no involvement in the study design, collection, analysis and interpretation of the data; or writing of the report.

In previous years Drs. Ortiz and Fernandez received research funding from Lilly S.A. Spain, Serono, Organon, and Pfizer.

Dr. Juan J. López-Ibor has received speaker's honoraria, travel grants or consultancy fees from Eli Lilly, Bristol-Myers Squibb, Lundbeck, Pfizer and Servier and is or has been member of advisory boards of Eli Lilly, Pfizer and Bristol-Myers Squibb and he is a faculty member of the Board of the Lundbeck Institute (Lundbeck Neuroscience Foundation) of Copenhaguen.

Dr. María Inés López-Ibor has received speaker's honoraria or travel grants from Eli Lilly, Bristol-Myers Squibb, Lundbeck and Servier.

In previous years Dr. Morón has received speaker's honoraria and travel grants from Bristol-Myers Squibb, Janssen-Cilagg Lundbeck, Boehringer-Ingelheim and Servier.

In previous years Dr. Matías-Santos has received funding from Pfizer, Eli Lilly, Jansen-Organon, and Wyeth.

The rest of authors have nothing to declare.

\section{Acknowledgement}

To Dr. Jamie Horder for his invaluable contribution to this manuscript.

\section{Appendix A. Lempel-Ziv complexity algorithm}

The detailed algorithm for the measure of the $L Z$ complexity is as follows (Zhang et al., 2001):

(1) Let $S$ and $Q$ denote two subsequences of the original sequence $P$ and $S Q$ be the concatenation of $S$ and $Q$, while $S Q \pi$ is a string derived from $S Q$ after its last character is deleted ( $\pi$ means the operation to delete the last character).

(2) Let $v(S Q \pi)$ denote the vocabulary of all different substrings of $S Q \pi$.

(3) At the beginning, the complexity counter $c(n)=1, S=s(1)$, $Q=s(2), S Q=s(1), s(2)$ and $S Q \pi=s(1)$.

(4) In general, suppose that $S=s(1), s(2), \ldots, s(r), Q=s(r+1)$ and, therefore, $S Q \pi=s(1), s(2), \ldots, s(r)$. If $Q \in \mathcal{U}(S Q \pi)$, then $Q$ is a subsequence of $S Q \pi$, not a new sequence.

(5) $S$ does not change and renew $Q$ to be $s(r+1), s(r+2)$, then judge if $Q$ belongs to $v(S Q \pi)$ or not.

(6) The steps 4 and 5 are repeated until $Q$ does not belong to $v(S Q \pi)$. Now $Q=s(r+1), s(r+2), \ldots, s(r+i)$ is not a subsequence of $S Q \pi=s(1), s(2), \ldots, s(r+i-1)$, so increase the counter by one.

(7) Thereafter, $S$ and $Q$ are combined and renewed to be $s(1)$, $s(2), \ldots, s(r+i)$, and $s(r+i+1)$, respectively.

(8) Repeat the previous steps until $Q$ is the last character. At this time, the number of different substrings is $c(n)$, the measure of complexity. 
In order to obtain a complexity measure which is independent of the sequence length, $c(n)$ should be normalised. If the length of the sequence is $n$ and the number of different symbols is $\alpha$, it has been proved (Lempel and Ziv, 1976) that the upper bound of $c(n)$ is given by:

$c(n)<\frac{n}{\left(1-\varepsilon_{n}\right) \log _{\alpha}(n)}$

where $\varepsilon_{n}$ is a small quantity and $\varepsilon_{n} \rightarrow 0(n \rightarrow \infty)$. In general, $n /$ lo$\mathrm{g}_{\alpha}(n)$ is the upper limit of $c(n)$, where the base of the logarithm is $\alpha$, i.e.,

$$
\lim _{n \rightarrow \infty} c(n)=b(n) \equiv \frac{n}{\log _{\alpha}(n)}
$$

For a binary conversion $\alpha=2$, and $b(n)$ is given by

$b(n) \equiv \frac{n}{\log _{2}(n)}$

and $c(n)$ can be normalised via $b(n)$ :

$C(n)=\frac{c(n)}{b(n)}$

$C(n)$ is usually a value between zero and one. The normalised $\mathrm{LZC}$ reflects the arising rate of new patterns along with the sequence (Zhang et al., 2001). A minimum data length must be considered to ensure that $L Z C$ reveals real data features (Yan and Gao, 2004). Since a previous work showed that the LZC values become stable for MEGs longer than 3000 samples (Gómez et al., 2006), an epoch length of 3392 data points (20 s) was used in the current study.

\section{References}

Aboy $M$, Hornero $R$, Abasolo D, Alvarez D. Interpretation of the Lempel-Ziv complexity measure in the context of biomedical signal analysis. IEEE Trans Biomed Eng 2006;53(11):2282-8.

Andreasen NC. The Scale for the Assessment of Positive Symptoms (SAPS). lowa City: University of lowa; 1984

Anokhin AP, Birbaumer N, Lutzenberger W, Nikolaev A, Vogel F. Age increases brain complexity. Electroencephalogr Clin Neurophysiol 1996;99(1):63-8.

Canive JM, Lewine JD, Edgar JC, Davis JT, Torres F, Roberts B, et al. Magnetoencephalographic assessment of spontaneous brain activity in schizophrenia. Psychopharmacol Bull 1996;32(4):741-50.

Cohen, J. Statistical power analysis for the behavioral sciences. 2nd ed. Hillsdale, NJ: Lawrence Earlbaum Associates; 1988.

Eckmann JP, Ruelle D. Fundamental limitations for estimating dimensions and Lyapunov exponents in dynamical systems. Physica D 1992;56(2-3):185-7.

Edgar JC, Miller GA, Moses SN, Thoma RJ, Huang MX, Hanlon FM, et al. Cross-modal generality of the gating deficit. Psychophysiology 2005;42(3):318-27.

Elbert T, Lutzenberger W, Rockstroh B, Berg P, Cohen R. Physical aspects of the EEG in schizophrenics. Biol Psychiatry 1992;32(7):595-606.

Fehr T, Kissler J, Wienbruch C, Moratti S, Elbert T, Watzl H, et al. Source distribution of neuromagnetic slow-wave activity in schizophrenic patients - effects of activation. Schizophr Res 2003;63(1-2):63-71.

Fernández A, Quintero J, Hornero R, Zuluaga P, Navas M, Gomez C, et al. Complexity analysis of spontaneous brain activity in attention-deficit/hyperactivity disorder: diagnostic implications. Biol Psychiatry 2009;65(7):571-7.

Fernández A, Hornero R, Gómez C, Turrero A, Gil-Gregorio P, Matías-Santos J, et al. Complexity analysis of spontaneous brain activity in Alzheimer's disease and mild cognitive impairment: a MEG study. Alz Dis Assoc Dis 2010;24(2):182-9.

First MB, Spitzer RL, Gibbon M, Williams JBW. Entrevista clínica estructurada para los trastornos del eje I del DSM-IV: SCID-I. New York: Columbia University; 1997.

Friston KJ. Theoretical neurobiology and schizophrenia. $\mathrm{Br}$ Med Bull $1996 ; 52(3): 644-55$.

Friston KJ. Dysfunctional connectivity in schizophrenia. World Psychiatry $2002 ; 1(2): 66-71$.

Gómez C, Hornero R, Abásolo D, Fernández A, López M. Complexity analysis of the magnetoencephalogram background activity in Alzheimer's disease patients. Med Eng Phys 2006;28(9):851-9.

Grassberger P, Procaccia I. Measuring the strangeness of strange attractors. Physica D 1983;9(1-2):189-208

Ho BC, Andreasen NC, Nopoulos P, Arndt S, Magnotta V, Flaum M. Progressive structural brain abnormalities and their relationship to clinical outcome: a longitudinal magnetic resonance imaging study early in schizophrenia. Arch Gen Psychiatry 2003;60(6):585-94.
Hosmer D, Lemeshow S. Applied logistic regression. New York: John Wiley and Sons, Inc; 1989.

Irisawa S, Isotani T, Yagyu T, Morita S, Nishida K, Yamada K, et al. Increased omega complexity and decreased microstate duration in nonmedicated schizophrenic patients. Neuropsychobiology 2006:54(2):134-9.

Itil TM. Qualitative and quantitative EEG findings in schizophrenia. Schizophr Bull $1977 ; 3(1): 61-79$.

Jeong J. EEG dynamics in patients with Alzheimer's disease. Clin Neurophysiol 2004;115(7):1490-505.

Jeong J, Kim DJ, Chae JH, Kim SY, Ko HJ, Paik IH. Nonlinear analysis of the EEG of schizophrenics with optimal embedding dimension. Med Eng Phys 1998;20(9):669-76.

Jones DK, Catani M, Pierpaoli C, Reeves SJC, Shergill SS, O'Sullivan MO, et al. Age effects on diffusion tensor magnetic resonance imaging tractography measures of frontal cortex connections in schizophrenia. Hum Brain Mapp 2006:27:230-8.

Kim DJ, Jeong J, Chae JH, Park S, Yong Kim S, Jin Go H, et al. An estimation of the first positive Lyapunov exponent of the EEG in patients with schizophrenia. Psychiatry Res 2000;98(3):177-89.

Kissler J, Muller MM, Fehr T, Rockstroh B, Elbert T. MEG gamma band activity in schizophrenia patients and healthy subjects in a mental arithmetic task and at rest. Clin Neurophysiol 2000;111(11):2079-87.

Kotini A, Anninos P. Detection of non-linearity in schizophrenic patients using magnetoencephalography. Brain Topogr 2002;15(2):107-13.

Koukkou M, Lehmann D, Wackermann J, Dvorak I, Henggeler B. Dimensional complexity of EEG brain mechanisms in untreated schizophrenia. Biol Psychiatry 1993;33(6):397-407

Krueger CE, Dean DL, Rosen HJ, Halabi C, Weiner M, Miller BL, et al. Longitudinal rates of lobar atrophy in frontotemporal dementia, semantic dementia, and Alzheimer's disease. Alzheimer Dis Assoc Disord 2010;24(1):43-8.

Lempel A, Ziv J. On the complexity of finite sequences. IEEE Trans Inform Theory $1976 ; 22(1): 75-81$.

Li Y, Tong S, Liu D, Gai Y, Wang X, Wang J, et al. Abnormal EEG complexity in patients with schizophrenia and depression. Clin Neurophysiol $2008 ; 119(6): 1232-41$.

Lieberman J, Chakos M, Wu H, Alvir J, Hoffman E, Robinson D, et al. Longitudina study of brain morphology in first episode schizophrenia. Biol Psychiatry 2001;49(6):487-99.

Lopez-Ibor JJ, Lopez-Ibor MI, Mendez MA, Moron MD, Ortiz-Teran L, Fernández A, et al. The perception of emotion-free faces in schizophrenia: a magnetoencephalography study. Schizophr Res 2008;98(1-3):278-86.

Lutzenberger $W$, Preissl $H$, Pulvermuller $F$. Fractal dimension of electroencephalographic time series and underlying brain processes. Biol Cybern 1995;73(5):477-82.

McGlashan TH. Is active psychosis neurotoxic? Schizophr Bull 2006;32(4):609-13.

McGlashan TH, Hoffman RE. Schizophrenia as a disorder of developmentally reduced synaptic connectivity. Arch Gen Psychiatry 2000;57(7):637-48.

Meyer-Lindenberg A. The evolution of complexity in human brain development: an EEG study. Electroencephalogr Clin Neurophysiol 1996;99(5):405-11.

Murray RM, Lewis SW. Is schizophrenia a neurodevelopmental disorder? Br Med J $1987 ; 295(6600): 681-2$

$\mathrm{Na} \mathrm{SH}$, Jin SH, Kim SY, Ham B]. EEG in schizophrenic patients: mutual information analysis. Clin Neurophysiol 2002;113(12):1954-60.

Naatanen R, Kahkonen S. Central auditory dysfunction in schizophrenia as revealed by the mismatch negativity (MMN) and its magnetic equivalent MMNm: a review. Int I Neuropsychopharmacol 2009;12(1):125-35.

Nagarajan R. Quantifying physiological data with Lempel-Ziv complexity - certain issues. IEEE Trans Biomed Eng 2002;49(11):1371-3.

Perez-Neri I, Ramirez-Bermudez J, Montes S, Rios C. Possible mechanisms of neurodegeneration in schizophrenia. Neurochem Res 2006:31(10):1279-94.

Rapoport JL, Addington AM, Frangou S, Psych MR. The neurodevelopmental mode of schizophrenia: update 2005. Mol Psychiatry 2005;10(5):434-49.

Roberts GW, Colter N, Lofthouse R, Bogerts B, Zech M, Crow T]. Gliosis in schizophrenia: a survey. Biol Psychiatry 1986;21:1043-50.

Roberts GW, Colter N, Lofthouse R, Johnstone EC, Crow TJ. Is there gliosis in schizophrenia? Investigation of the temporal lobe. Biol Psychiatry 1987:22:1459-68.

Saito N, Kuginuki T, Yagyu T, Kinoshita T, Koenig T, Pascual-Marqui RD, et al. Global, regional, and local measures of complexity of multichannel electroencephalography in acute, neuroleptic-naive, first-break schizophrenics. Biol Psychiatry 1998;43(11):794-802.

Shagass C, Roemer RA, Straumanis JJ. Relationships between psychiatric diagnosis and some quantitative EEG variables. Arch Gen Psychiatry 1982;39(12):1423-35.

Shaw P, Eckstrand K, Sharp W, Blumenthal J, Lerch JP, Greenstein D, et al. Attentiondeficit/hyperactivity disorder is characterized by a delay in cortical maturation. Proc Natl Acad Sci 2007:104(49):19649-54.

Sporns O, Tononi G, Edelman GM. Connectivity and complexity: the relationship between neuroanatomy and brain dynamics. Neural Netw 2000;13(89):909-22.

Theberge J, Williamson KE, Aoyama N, Drost DJ, Manchanda R, Malla AK, et al. Longitudinal grey-matter and glutamatergic losses in first-episode schizophrenia. Br J Psychiatry 2007;191:325-34.

Tononi G, Edelman GM, Sporns O. Complexity and coherency: integrating information in the brain. Trends Cogn Sci 1998:2(12):474-84. 
Tully E, McGlashan TH. The prodrome. In: Lieberman JA, Stroup TS, Perkins DO, editors. Textbook of schizophrenia. Arlington: American Psychiatric Publishing; 2006. p. 341-52.

Wienbruch C, Moratti S, Elbert T, Vogel U, Fehr T, Kissler J, et al. Source distribution of neuromagnetic slow wave activity in schizophrenic and depressive patients. Clin Neurophysiol 2003;114(11):2052-60.
Woods BT. Is schizophrenia a progressive neurodevelopmental disorder? Toward a unitary pathogenetic mechanism. Am J Psychiatry 1998;155(12):1661-70.

Yan R, Gao RX. Complexity as a measure for machine health evaluation. IEEE Trans Instrum Meas 2004;53(4):1327-34.

Zhang XS, Roy RJ, Jensen EW. EEG complexity as a measure of depth of anesthesia for patients. IEEE Trans Biomed Eng 2001;48(12):1424-33. 